\title{
A Front Tracking Method Based on Runge-Kutta Discontinuous Galerkin Methods
}

\author{
https://doi.org/10.3991/ijoe.v12i12.6453 \\ Xu-jiu Zhang ${ }^{1 *}$,Yong-sheng Zhu ${ }^{1}$, Ke Yan ${ }^{1,2}$, You-yun Zhang ${ }^{1}$ \\ ${ }^{1} \mathrm{Xi}$ 'an Jiaotong University, Xi'an, China; \\ ${ }^{2}$ Concordia University, Montreal, Canada.
}

\begin{abstract}
In this paper, a high-resolution front tracking method was presented for interface tracking simulation with Runge-Kutta discontinuous Galerkin methods. An interface treating method of the discontinuous methods is presented. This method don't construct the ghost fluid and the flow information on both sides next to the interface is used to solve the interfacial status. The limiter adopted the combination of the shock detection and monotonicity-preserving limiter and level set method is used for tracking the interface. Result shown that the front tracking of the high-order accurate Runge-Kutta discontinuous Galerkin method exhibits very good agreement with exact solution in the interface condition that contain strong shock.
\end{abstract}

IndexTerms-Runge-Kutta discontinuous Galerkin methods; high-resolution; limiter; monotonicity-preserving schemes; level set technique.

\section{INTRODUCTION}

The treatment of moving interfaces and their vicinity field is crucial in multi-medium flow simulation due to its discontinuous contact. In general, these types of methods can be separated into two categories by how each considers the interfaces: diffuse interface method (DIM) and sharp interface method (SIM).

In the DIM ${ }^{[9,10,11,12]}$, the interface is modeled as a numerically diffused one (area), which is similar to capturing a discontinuity in gas dynamics. In fact, it can be mentioned that this type of diffused interface is a kind of artificial diffusion that is created by numerical calculations. A way to circumvent the numerical diffusion is to use a higher order method. The major drawback in diffuse interface methods is the numerical diffusion which can lead to a very bad representation of the interfaces, especially when long time computations are needed.

In the sharp interface methods, a special effort is made to find the right location of the interface and to treat the interface explicitly. This method includes Euler methods, including the level set ${ }^{[14]}$ and VOF approaches ${ }^{[13]}$ combined Euler-Lagrangi-an methods, including front tracking and front tracking with ghost fluid methods, and ALE methods ${ }^{[15]}$. ALE methods often result in strong distorted meshes. the mesh must be able to capture all inclusions, which may be very costly if these ones are small.

The other important part is the interface treating method. The GFM ${ }^{[1,2,3,4,5,6,7,8]}$ (ghost fluid method) treats the material interface as an internal boundary, and by defining ghost cells and ghost fluids, the two-medium flow can be solved via two respective single-medium Riemann prob- lems. Based on the GFM, a new interface treating method is presented.

This article is concerned with the approximation of multiphase compressible flows with a high order method. We will only deal with interface problems and are interested in discontinuous Galerkin (DG) methods and level set method ${ }^{[19-20]}$ is used for tracking the interface.

The completely discontinuous piecewise polynomial space is employed in DG method, which was used for solving the first order linear and nonlinear hyperbolic problem since the discontinuous Galerkin finite method was proposed by Reed and Hill ${ }^{[7]}$ for neutron transport equations. Cockbum et al. established a framework for nonlinear time dependent hyperbolic conservation law, using nonlinearly stable high order Runge-Kutta time discretization ${ }^{[8]}$, discontinuous Galerkin discretization in space with Riemann solvers as numerical fluxes and total variation bounded nonlinear limiter ${ }^{[9]}$, to achieve nonoscillatory properties in condition of strong shocks. There are many limiters ${ }^{[14,22,23,24]}$, and in this article, the limiter adopted the combination of the shock detection and monotonicity-preserving limiter.

\section{Governing Equations And NumericAl Methods}

\section{A. Governing Equation}

A $1 D$ analysis is given for illustrating the alghrothm in certain situation. The 1D Euler equation gives as,

$$
\frac{\partial U}{\partial t}+\frac{\partial F(U)}{\partial x}=0
$$

Where $U=[\rho, \rho u, E] T, F(U)=[\rho u, \rho u 2+p,(E+p) u] T, \rho$ is the density, $u$ is the velocity, $p$ is the pressure and $E$ is the total energy. The total energy $\mathrm{E}$ is given as follow:

$$
\mathrm{E}=\rho \mathrm{e}+\rho \mathrm{u} 2 / 2
$$

Where e is the specific internal energy. For system closure purpose, the equation of state (EOS) is needed. Assuming the EOS here is equivalent to the Mie-Gruneison family state equations, then one obtains:

$$
\rho e=f(\rho) p+g(p)
$$

Where $\mathrm{f}$ and $\mathrm{g}$ are functions of density and some other constants relative to heat conductivity. Here we focus on two types of EOS. The first one is the $\gamma$-law for perfect gases, in this case $f=1 /(\gamma-1)$ and $g=0.0$. The second one is the Tait's EOS for water, where $\mathrm{f}=1 /(\mathrm{N}-1)$ and $\mathrm{g}=$ $\mathrm{N}(\mathrm{B}-\mathrm{A}) /(\mathrm{N}-1), \mathrm{N}=7.15, \mathrm{~A}=1.0 \times 105 \mathrm{~Pa}, \mathrm{~B}=3.31 \times 108 \mathrm{~Pa}$ 
and $\rho=1.0 \times 103 \mathrm{~kg} / \mathrm{m} 3$. Detail of the two types of EOS can be seen in Ref. [3].

\section{B. The Interface Treating Method}

In this section, present the new interface treating method.

The original GFM, is shown in Fig. 1 (a) and An illustration of the real GFM procedure ${ }^{[21]}$ is shown in Fig. 1 (b). the information of the neighbor cell is employed to update the interface status. In the mixed cell method, the mixed cell is employed for predicting the interfacial status and the predicted status update the fluid flow states in the interface element.

In this paper, we employ the data at the centroid of the neighbor cell of the interface element to define the Riemann problem at the interface for predicting the interfacial status. An illustration is shown in Fig. 1 (c)

Assume Ii $=[x i-1 / 2, x i+1 / 2]$ is the cell where the interface locates and is called the interface cell. The cells Ii-1 and $\mathrm{I}+1$ are single medium cells as shown in Fig. 1(c). A Riemann problem can be constructed by taking the input data as $\mathrm{UL}=\mathrm{Uhi}-1$ and $\mathrm{UR}=\mathrm{Uhi}+1 \quad(\mathrm{UL}=\mathrm{Uhi}$ and $\mathrm{UR}=\mathrm{Uhi}+1$, or $\mathrm{UL}=\mathrm{Uhi}-1$ and $\mathrm{UR}=\mathrm{Uhi}+2)$. The intermediate states of the interface are $\mathrm{UIL}=(\rho \mathrm{IL}, \mathrm{uI}, \mathrm{pI})$ and $\mathrm{UIR}=(\rho I R, u I, p I)$.

In the process of iteration, the numerical procedure we use is the following:

1. To carry out a time marching, Eq.(1) is solved using the third Runge-Kutta scheme and a time step is selected to satisfy the CFL condition.

2. Advance and reinitialize the level set function field

3. Find the interface cell $\left(\varphi_{i+\frac{1}{2}} \times \varphi_{i-\frac{1}{2}} \leq 0\right)$ and construct the Riemann solver. One get the intermediate state.

4. Update $U$ according to the DG method except the boundary. When the neighboring cell is the interface cell, the source term in the DG method is selected as follows

$$
\begin{cases}(f)_{N B}=f\left(\rho_{I L}, u_{I}, p_{I}\right),(U)_{N B}=f\left(\rho_{I L}, u_{I}, p_{I}\right), & \varphi>0 \\ (f)_{N B}=f\left(\rho_{I R}, u_{I}, p_{I}\right),(U)_{N B}=f\left(\rho_{I R}, u_{I}, p_{I}\right), & \varphi<0\end{cases}
$$

5. Carry out the limiter to control the numerical oscillations.

6. Repeat the step 4 and step 5until the third RungeKutta scheme is achieved

7. Repeat the step 1-6 until the calculation is achieved.

When the above procedure is employed to calculate, we find some classic limiter is not as efficient as in the past and make some improvement.

\section{NUMERICAL EXPERIMENTS}

Numerical experiments will be carried out for the investigation of the presented interface treating method and the monotonicity-preserving limiter. All example adopts the level set method to track the interface. Here mGFM1 denotes the real ghost method by taking the input data as $\mathrm{UL}=\mathrm{Uhi}$ and $\mathrm{UR}=\mathrm{Uhi}+1$, and $\mathrm{mGFM} 2$ denotes the presented interface treating method. Gas shock tube problem were simulated for test the method.

Consider a 2-m domain with 2000 grid cells. The domain is filled with kind of compressible flow. The initial

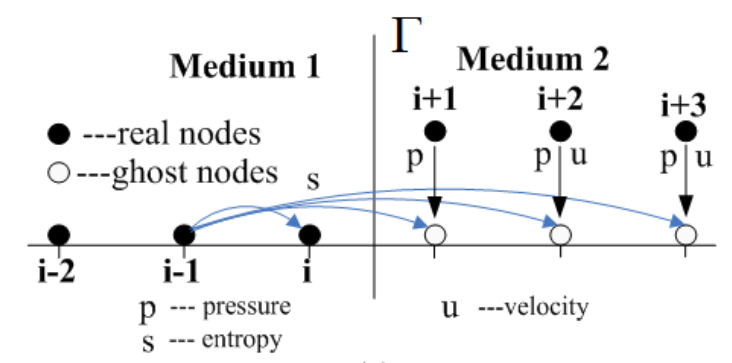

(a)

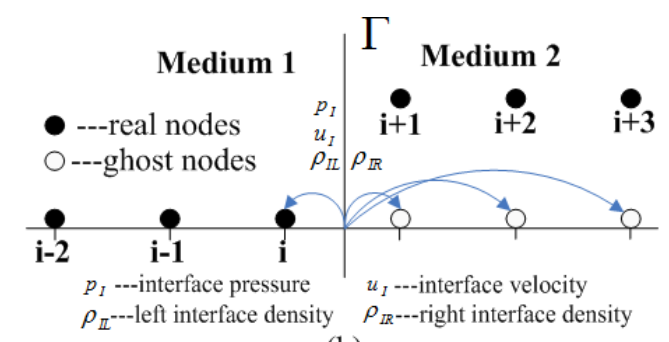

(b)

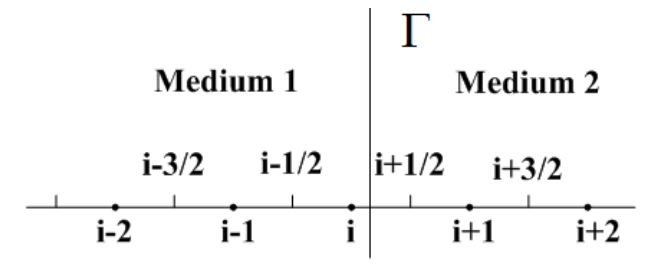

(c)

Figure 1. Updating the real and ghost nodes in the ghost fluid methods and the presented methods

flow parameters on both sides of the interface are $\mathrm{pH}=$ $1.0, \mathrm{pL}=0.1, \rho \mathrm{H}=1.0, \rho \mathrm{L}=0.125, \mathrm{uH}=\mathrm{uL}=0.0, \gamma \mathrm{H}=$ $\gamma \mathrm{L}=1.4, \mathrm{CFL}=0.3$. The interface is initially located at $\mathrm{x} 0$ $=1.0$. the calculation time is $0.4 \mathrm{~s}$.

Here the presented interface treating method is employed for testing accurate monotonicity -preserving limiter.

Pressure and density of the computation are shown in Fig. 2 (a1-a2) when the monotonicity -preserving limiter was employed for detection and reconstruction. Obviously, oscillation occurs in rarefaction wave region. So we make an improvement. The shock detection is adopter for discontinuity detection and monotonicity-preserving limiter for reconstruction is just for reconstruction. The result is shown in Fig. 2(b1-b2). We can see that the result is optimal and this scheme can inhibit the oscillation.

\section{CONCLUSION}

In this paper, the front tracking with Runge-Kutta discontinuous Galerkin methods was presented.

The level set method is used to track the interface. The aim of this research focuses on the interface treating method and numerical oscillation suppression. The conclusions are as follow:

The presented method can work well when the Riemann problem can be constructed and implementation of the method is easier compared to the real ghost fluid method. For numerical oscillation suppression, considerable accuracy was achieved when the combination of shock detection and the monotonicity-preserving was employed. 


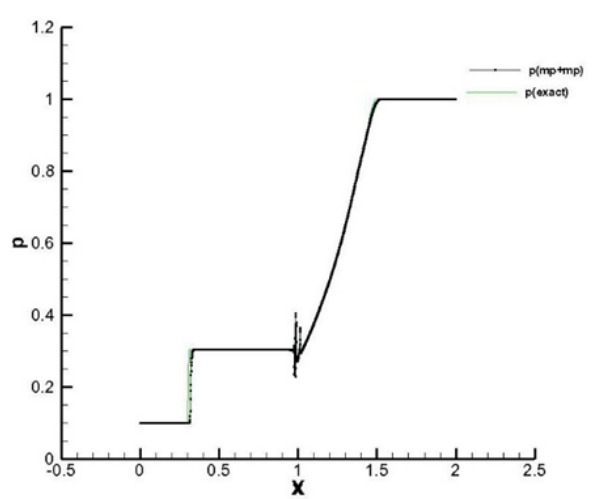

(a1)
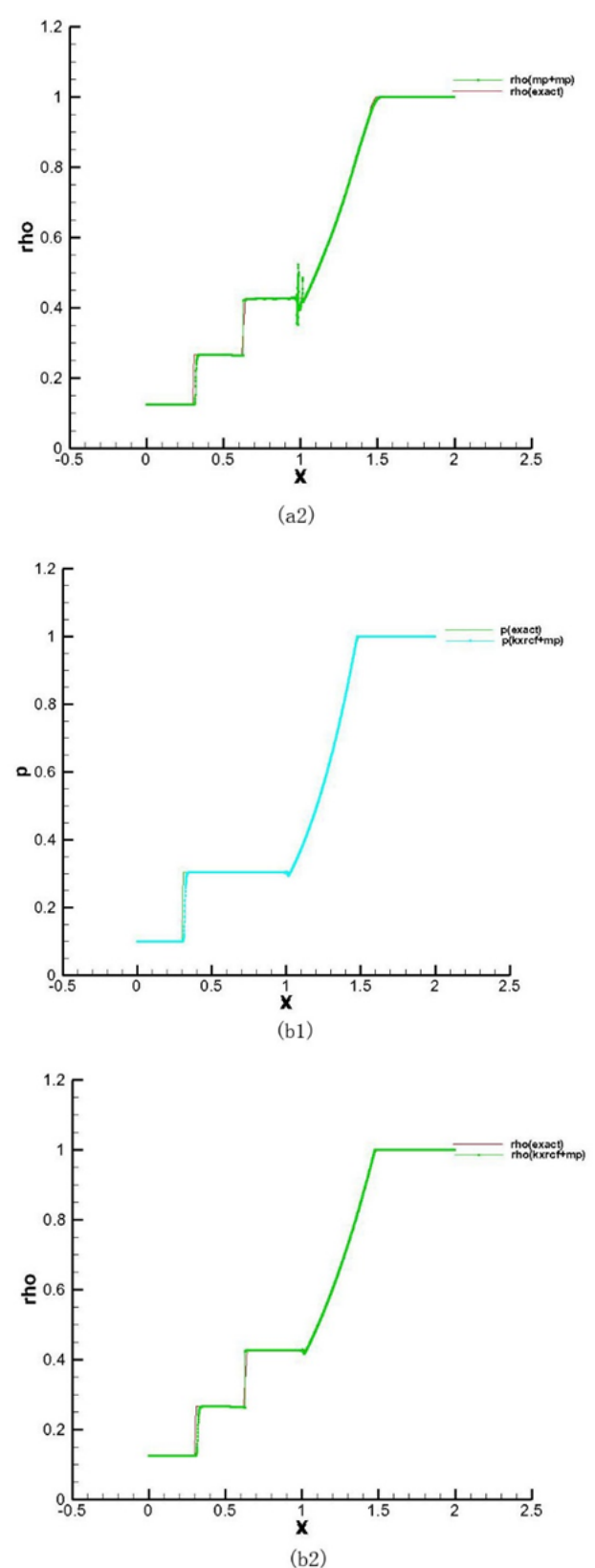

Figure 2. Comparison of computation data to the analytical results (a1) Pressure obtained when mp is used to detect and restructure; (a2) Density obtained when mp is used to detect and restructure; (b1) Pressure obtained when kxrcf is used to detect and $\mathrm{mp}$ is used to restructure; (b2) Density obtained when kxrcf is used to detect and $\mathrm{mp}$ is used to restructure.

\section{REFERENCES}

[1] R.P. Fedkiw, A. Marquina, B. Merriman. An isobaric fix for overheating problem in multimaterial compressible flows. Journal of Computation Physics, 1999, 148(2): 545-578. https://doi.org/10.1006/jcph.1998.6129

[2] Duc Nguyen, Frédéric Gibou, Ronald Fedkiw. A Fully Conservative Ghost Fluid Method Stiff Detonation Waves. 2002 .In 12th Int. Detonation Symposium

[3] T.G.Liu, B.C. Khoo, K.S. Yeo. Ghost fluid method for strong shock impacting on material interface. Journal of Computation Physics, 2003, 190(2): 651-681. https://doi.org/10.1016/S00219991(03)00301-2

[4] R.P. Fedkiw, T. Aslam, B. Merriman,et al. A non-oscillatory Eulerian approach to interfaces in multimaterial flows (the Ghost Fluid Method). Journal of Computation Physics, 1999, 152(2): 457-492. https://doi.org/10.1006/jcph.1999.6236

[5] C. Wang, C.W. Shu. An interface treating technique for compressible muti-medium flow with Runge-Kutta discontinuous Galerkin method. Journal of Computation Physics, 2010, 229(23): 88238843. https://doi.org/10.1016/j.jcp.2010.08.012

[6] J.X. Qiu, T.G. Liu, B.C. Khoo. Runge-kutta discontinuous Galerkin methods for compressible two-medium flow simulations: one dimensional case. Journal of Computation Physics, 2007, 222(1): 353-373. https://doi.org/10.1016/j.jcp.2006.07.023

[7] J.X. Qiu, T.G. Liu, B.C. Khoo. Runge-kutta discontinuous Galerkin methods with the ghost fluid method. Communications in Computational Physics, 2008, 3: 479-504.

[8] Ryan W. Houim, Kenneth K. Kuo .A ghost fluid method for compressible reacting flows with phase change. Journal of Computational Physics, 2013,235: 865-900 https://doi.org/10.1016/ j.jcp.2012.09.022

[9] Erwin Franquet, Vincent Perrier. Runge-Kutta discontinuous Galerkin method for the approximation of Baer and Nunziato type multiphase models. Journal of Computational Physics,2012 231: 4096-4141 https://doi.org/10.1016/j.jcp.2012.02.002

[10] Tao Xiong, Chi-Wang Shu, Mengping Zhang. WENO Scheme with Subcell Resolution for Computing Nonconservative Euler Equations with Applications to One-Dimensional Compressible Two-Medium Flows. J Sci Comput, 2012, 53:222-247 https://doi.org/10.1007/s10915-012-9578-7

[11] Erwin Franquet, Vincent Perrier. Runge-Kutta discontinuous Galerkin method for interface flowswith a maximum preserving limiter. Computers \& Fluids, 2012, 65: 2-7 https://doi.org/10.1016/j.compfluid.2012.02.021

[12] M.R. Ansari, A. Daramizadeh. Numerical simulation of compressible two-phase flow using a diffuse interface method. International Journal of Heat and Fluid Flow, 2013, 42: 209-223 https://doi.org/10.1016/j.ijheatfluidflow.2013.02.003

[13] Pilliod, J., Puckett, E.. Second-order accurate volume-of-fluid algorithms for tracking material interfaces. J. Comput. Phys,2004, 199: 465-502. https://doi.org/10.1016/j.jcp.2003.12.023

[14] Osher, S., Fedkiw, R. 2001. Level set methods: an overview and some recent results.J. Comput. Phys, 2001,169 (2), 463-502. https://doi.org/10.1006/jcph.2000.6636

[15] Anbarlooei, H.R., Mazaheri, K. 2009. Moment of fluid interface reconstruction method in multi-material arbitrary Lagrangian Eulerian (MMALE) algorithms. Comput. Meth. Appl. Mech. Eng, 2009,198, 3782-3794. https://doi.org/10.1016/j.cma.2009.08.009

[16] W.H Reed and T.R.Hill(1973). Triangular mess methods for neutron transport equation. Technical report LA-UR-73-476T Los Alamos scientific Laboratory $\mathrm{T}$

[17] C.W. Shu, S. Osher. Efficient implementation of essentially nonoscillatory shock capturing schemes. Journal of Computation Physics, 1988, 77(2): 439-471. https://doi.org/10.1016/00219991(88)90177-5

[18] B. Cockburn, C. W. Shu. TVB Runge-Kutta local projection discontinues Galerkin finite elelment method for conservation laws II: general framework. Mathematics of Computation, 1989, 52: 411-435.

[19] S. Osher, J.A. Sethian. Fronts propagating with curvaturedependent speed: algorithms based on Hamilton-Jacobi formulations. Journal of Computation Physics, 1988, 79(1): 12-49. https://doi.org/10.1016/0021-9991(88)90002-2 


\section{A Front Tracking Method Based on Runge-Kutta Discontinuous Galerkin Methods}

[20] D. Adalsternsson, J.A. Sethian. A fast level set method for propagating interface. Journal of Computation Physics, 1995, 118(2): 269-277. https://doi.org/10.1006/jcph.1995.1098

[21] C. W. Wang, T. G. Liu, And B. C. Khoo. A Real Ghost Fluid Method For The Simulation Of Multimedium Compressible Flow. Siam J. Sci. Comput, 2006, Vol. 28, No. 1, pp. 278-302 https://doi.org/10.1137/030601363

[22] C.W. Shu. TVB uniformly high-order schemes for conservation laws. Mathematics of Computation, 1987, 49: 105-121. https://doi.org/10.1090/S0025-5718-1987-0890256-5

[23] A. Suresh, H.T. Huynh. Accurate monotonicity-preserving schemes with Runge-Kutta time stepping. Journal of Computation Physics, 1997, 136(1): 83-99. https://doi.org/10.1006/jcph. 1997.5745

[24] L. Krivodonova, J. Xin, J.F. Remacle, et al. Shock detection and limiting with discontinuous Galerkin methods for hyperbolic conservation laws. Applied Numerical Mathematics, 2004, 48(3-4): 323-338. https://doi.org/10.1016/j.apnum.2003.11.002

[25] H. Luo, J.D. Baum. On the computation of steady-state compressible flows using a discontinuous Galerkin method. International Journal for Numerical Methods in Engineering, 2008, 73(5): $597-$ 623. https://doi.org/10.1002/nme.2081

\section{AUTHORS}

Xu-jiu Zhang is with the Key Laboratory of Education Ministry for Modern Design and Rotor-bearing System, Xi'an Jiaotong University, Xi'an, 710049, China (e-mail: zhangxuj@stu.xjtu.edu.cn).

Yong-sheng Zhu is with the Key Laboratory of Education Ministry for Modern Design and Rotor-bearing System, Xi'an Jiaotong University, Xi'an, 710049, China.

Ke Yan is with the Key Laboratory of Education Ministry for Modern Design and Rotor-bearing System, Xi'an Jiaotong University, Xi'an, 710049, China, and with the Department of Mechanical and Industrial Engineering, Concordia University, Montreal H3G 1M8, Canada.

You-yun Zhang is with the Key Laboratory of Education Ministry for Modern Design and Rotor-bearing System, Xi'an Jiaotong University, Xi'an, 710049, China.

The project was financially supported by the National Key Basic Research and Development Program (973 Program) (No. 2011CB706606), the National Natural Science Foundation of China (Grant No. 51405375) and the Bourses d'excellence pour étudiants étrangers du MESRS of Quebec, Canada (Programme 2C, Groupe 1, 188022). Submitted 26 October 2016. Published as resubmitted by the authors 27 November 2016. 